\title{
Zur Theorie des Belebtschlammverfahrens
}

II. Über den Mecbanismus der Elimination gelöster, organischer Stoffe aus Abwasser bei der biologischen Reinigung.

Von K. WUHRMANN und F. v. BEUST

Mitteilung Nr. I46 aus der Eidg. Anstalt für Wasserversorgung, Abwasserreinigung und Gewässerschutz an der Eidgenössischen Technischen Hochschule, Zürich

Direktor: Prof. Dr. O. JAAG

\section{Einleitung}

Wird die biologische Reinigung eines organisch verunreinigten Abwassers mittels summarischer Konzentrationsparameter für die Schmutzstoffe (zum Beispiel BSB 5 , Oxydierbarkeit, organischer Stickstoff usw.) verfolgt, so findet man eine exponentiell verlaufende Konzentrationsabnahme der Schmutzstoffe (vgl. zum Beispiel RIDENOUR [II $]^{1}$ ), GELLMAN und HeUKELEKIAN [6], Hoover et al. [7]). Es setzte sich deshalb die Auffassung fest (auch nach Überwindung der rein mechanistischen, chemisch-physikalischen Anschauungen von THÉRIAUlT [I4, I5] über den Mechanismus der Abwasserreinigung mittels Belebtschlamm), dass die Aufarbeitung eines Abwassers in zwei deutlich zu unterscheidenden Stufen erfolge: I. Adsorption der Abwasserstoffe an die Belebtschlammflocken und 2. anschliessender allmählicher Abbau der festgehaltenen Partikel durch die Organismen des Schlammes. Die Experimente von RucHHOFT et al. [12] ergaben erneut eine wichtige Stütze für diese Auffassung, die in allen Lehrbüchern und Abhandlungen als Grundlage des Belebtschlammverfahrens dargestellt wird. Nun haben wir aber in unserer früheren Arbeit (WUHRMANN et al. [I7]) gezeigt, dass innerhalb der analytisch heute zugänglichen Konzentrationsbereiche eine Reihe organischer Substanzen von Belebt-

1) Die Ziffern in eckigen Klammern verweisen auf das Literaturverzeichnis, Seite 329. 
schlammsuspensionen mit konstanter Geschwindigkeit aus der Lösung aufgenommen wird. Suspendiert man Belebtschlamm in einem Lösungsgemisch verschiedener reiner Substanzen, so nimmt auch dann die Konzentration jeder einzelnen Komponente linear mit der Kontaktzeit ab. Prüft man dagegen die « Konzentration» eines solchen Gemisches mit Hilfe seines BSB, so tritt eine exponentiell verlaufende Abnahme dieses Parameters in Funktion der Zeit ein, wie er von den zitierten amerikanischen Autoren beschrieben wurde.

Wir haben gezeigt, dass dieser exponentielle Reaktionsverlauf lediglich durch das Zusammenwirken der für jede vorhandene Substanz charakteristischen, konstanten Eliminationsgeschwindigkeit zustande kommt. Es fragt sich deshalb, ob unter diesen Umständen die bisherigen Auffassungen über den Mechanismus des Reinigungsvorganges aufrecht erhalten werden können, denn eine lineare Konzentrationsabnahme von gelösten Stoffen aus dem Abwasser lässt sich mit Adsorptionsvorgängen, die im Experiment mindestens annäherungsweise einen Verlauf der Eliminationskurve gemäss der Freundlichschen Adsorptionsisotherme ergeben müssten, nicht vereinbaren. Wir geben nachstehend die Ergebnisse von Beobachtungen bekannt, die einen Beitrag zur Kenntnis der bei der biologischen Abwasserreinigung wirksamen Mechanismen liefern sollen, unter Berücksichtigung der Kinetik der Elimination gelöster, organischer Stoffe, wie sie in unserer früheren Arbeit dargestellt wurde.

Arbeitsbypotbese: Die Dauer eines erhöhten Respirationsstoffwechsels von Belebtschlamm nach Zusatz eines geeigneten Substrates stellt innerhalb gewisser Grenzen einen Maßstab für die Zeitperiode dar, während welcher das Substrat den Mikroorganismen in der Lösung noch zurVerfügung steht. Verfolgt man mit der Messung der Atmungssteigerung gleichzeitig auf analytischem Wege die Konzentration des Substrates in der Schlammsuspension, so muss sich sofort feststellen lassen, in welcher Weise die Konzentrationsabnahme in der Lösung mit der Dauer und Intensität der Substratrespiration zusammenhängt. Spielen Adsorptionsvorgänge eine wesentliche Rolle bei der Elimination gelöster, organischer Substanzen des Abwassers, so sollte ein Substrat aus einem Schlammgemisch verschwunden sein, lange bevor die Substratatmung der Schlammorganismen dem Nullwert zustrebt. Ferner müsste die Konzentrationsabnahme des Substrates einen exponentiellen, die Atmungssteigerung dagegen in weitem Bereich einen linearen Verlauf nehmen (abgesehen von Fällen von Substratadaptation, bei welchen die Geschwindigkeit der Substratatmung und auch der Elimination mit der Zeit zunimmt). 


\section{Methoden}

Alle Experimente erfolgten mit Belebtschlamm (aus der Gemeindekläranlage Pfäffikon $\mathrm{ZH}$ oder aus unseren Versuchsanlagen), der für die Versuche in der früher beschriebenen Weise vorbereitet wurde. Je $3 \mathrm{~cm}^{3}$ gewaschener Schlamm (suspendiert in Phosphatpuffer, in der Regel o,005 $\mathrm{m}$, $\mathrm{pH} 7,5)$ wurden in die Gefässe von Barcroft-Mikromanometern ( $\mathcal{F}=$ rund $40 \mathrm{~cm}^{3}$ ) einpipettiert und in einem Seitenarm $\mathrm{I} \mathrm{cm}^{3}$ der Substratlösung vorgelegt. Im allgemeinen wurde sowohl der Sauerstoffverbrauch wie auch die $\mathrm{CO}_{2}$-Produktion gemessen ("direkte Methode» mit 2 Manometern, vgl. Dixon [5]). Da I6 Manometer zurVerfügung standen, wurden die Versuche wie folgt organisiert: 2 oder 4 Manometer wurden für die Messung der endogenen Atmung $\left(\mathrm{O}_{2}\right.$ und $\left.\mathrm{CO}_{2}\right)$ reserviert (Kipplösung $=$ destilliertes Wasser). I2 bis I4 Manometer waren für die Messung der Substratatmung bestimmt. Unmittelbar nach Schliessen der Manometer wurde die Substratlösung zum Schlamm gekippt. Nach bestimmten Zeitabständen wurden die Beobachtungen gleichzeitig bei 2 oder 4 Manometern (je I oder 2 für $\mathrm{O}_{2}$-bzw. $\mathrm{CO}_{2}$-Messung) abgebrochen, die Schlammsuspension aus den Gefässen auspipettiert, scharf abzentrifugiert und die klaren, überstehenden Lösungen für die analytische Bestimmung der verbliebenen Substratmengen vorbereitet. Auf diese Weise ergaben sich 2- oder 4 fache Substratbestimmungen pro Atmungsperiode. Die Präzision der Messungen des Gasumsatzes in den Manometern hielt sich im üblichen Rahmen ( $\mathrm{I}-2 \%$ Differenz zwischen Doppelbestimmungen). Sämtliche Versuche erfolgten bei $25^{\circ} \mathrm{C}$.

Für die Substratanalysen wurden die in der früheren Arbeit bereits erwähnten Methoden verwendet [I7].

\section{Versuchsergebnisse}

\section{a) Elimination von Zuckern}

Abbildung I a gibt die vollständigen Daten des Experimentes $53 / 7 / 3$ mit Belebtschlamm aus einer Anlage wieder, die während mehrerer Wochen ein Abwasser mit einem Saccharosegehalt von $1500 \mathrm{mg} / 1$ reinigte (Raumbelastung der Anlage $\mathrm{I}_{4} \mathrm{~m}^{3} / \mathrm{m}^{3}$. Tag). Der Schlamm war also zur Zeit des Manometerversuches vollständig an Saccharose adaptiert. Ein Vergleich der Kurven für die Konzentrationsabnahme des Substrates mit der Kurve für die reine Substratatmung zeigt sofort, dass die Saccharose aus der 


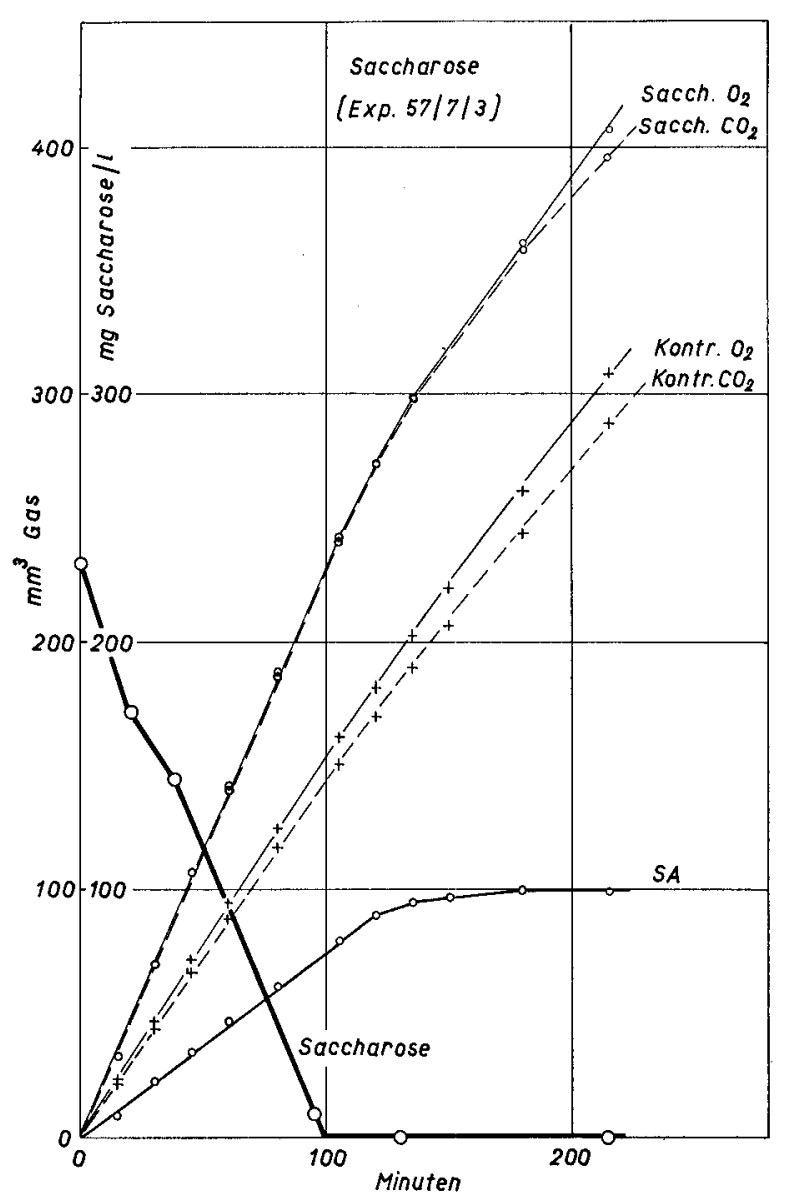

Abbildung 1 a

Sauerstoffverbrauch und Kohlensäureproduktion von Belebtschlamm während der Elimination von Saccharose. SchIamm an Saccharose adaptiert. Bezeichnungen: Sacch. $\mathrm{O}_{2}$ bzw. Sacch. $\mathrm{CO}_{2}$ $=$ Gasumsatz mit Saccharose (Substrat-Atmung). Kontr. $\mathrm{O}_{2}$ bzw. Kontr. $\mathrm{CO}_{2}=$ Gasumsatz. desselben Schlammes ohne Substrat (endogene Atmung). $S A=$ zusätzlicher Sauerstoffverbrauch wegen Substratzusatz. Kurve "Saccharose" = Substratkonzentration im Respirationsgefäss während des Atmungsversuches.

Schlammsuspension etwa 20 min vor dem Erreichen der endogenen Atmung eliminiert war. Der praktisch lineare Verlauf der Zuckerkonzentration schliesst aber aus, dass Adsorptionsvorgänge an den Schlammflocken massgebend am Verschwinden des Substrates beteiligt sein konnten. Der Zucker wurde offenbar ausschliesslich auf resorptivem Wege von den 
Schlammorganismen aus der Lösung aufgenommen, wobei die Resorption aber schneller verlief als die oxydative Dissimilation.

Aus dem in Abbildung $\mathrm{x} b$ dargestellten Experiment mit einem gewöhnlichen Belebtschlamm, dem Glukose zugesetzt wurde, geht dieselbe Erscheinung hervor: Die Zuckerkonzentration nimmt ungefähr proportional zur Kontaktzeit ab, und der Zucker verschwindet wiederum merklich vor dem Zeitpunkt der Wiederherstellung der ursprünglichen Atmungsintensität aus der Lösung.

Als weiteres Beispiel aus der Reihe unserer zahlreichen Versuche seien schliesslich noch die Beobachtungen über die Veratmung von Glukose durch einen gewöhnlichen, nicht adaptierten Schlamm, dem gleichzeitig

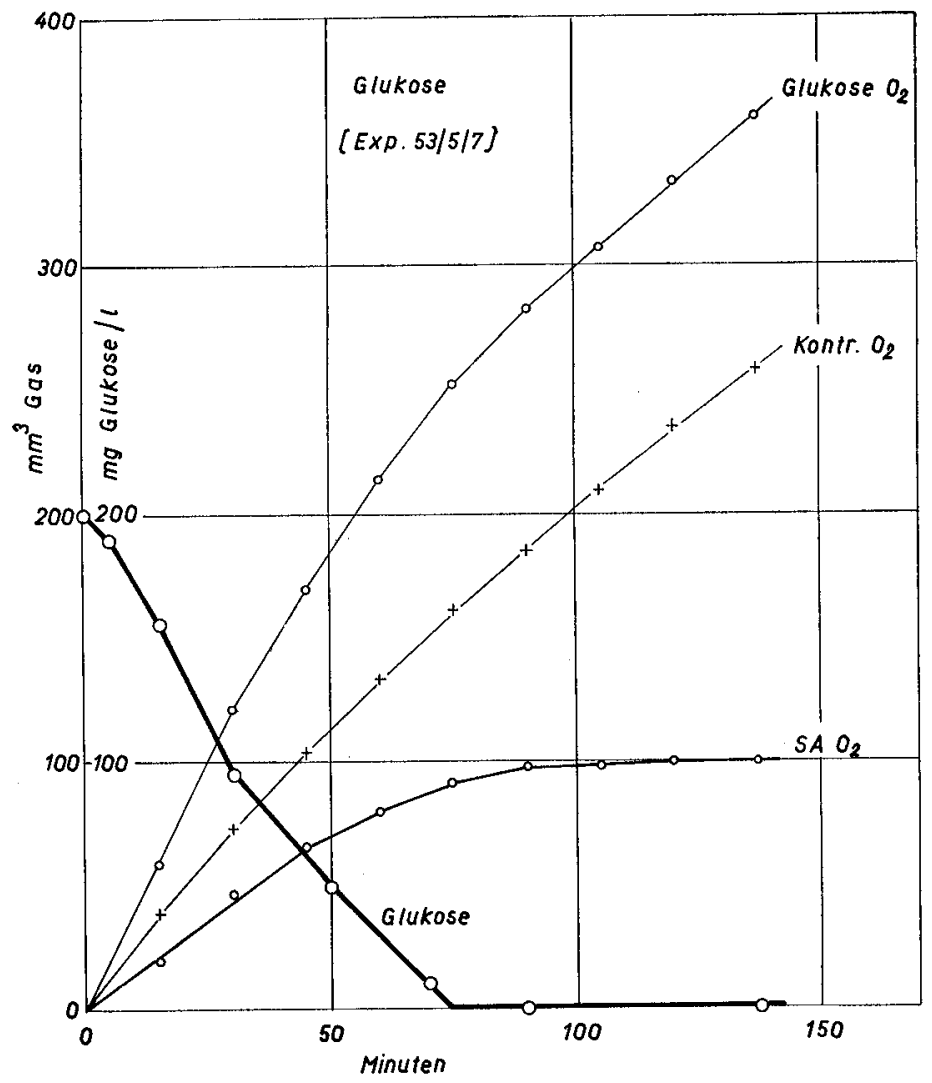

Abbildung $1 b$

Sauerstoffverbrauch von Belebtschlamm während der Elimination von Glukose. Schlamm nicht an Substrat adaptiert. Bezeichnungen sinngemäss wie in Abbildung 1. a. 


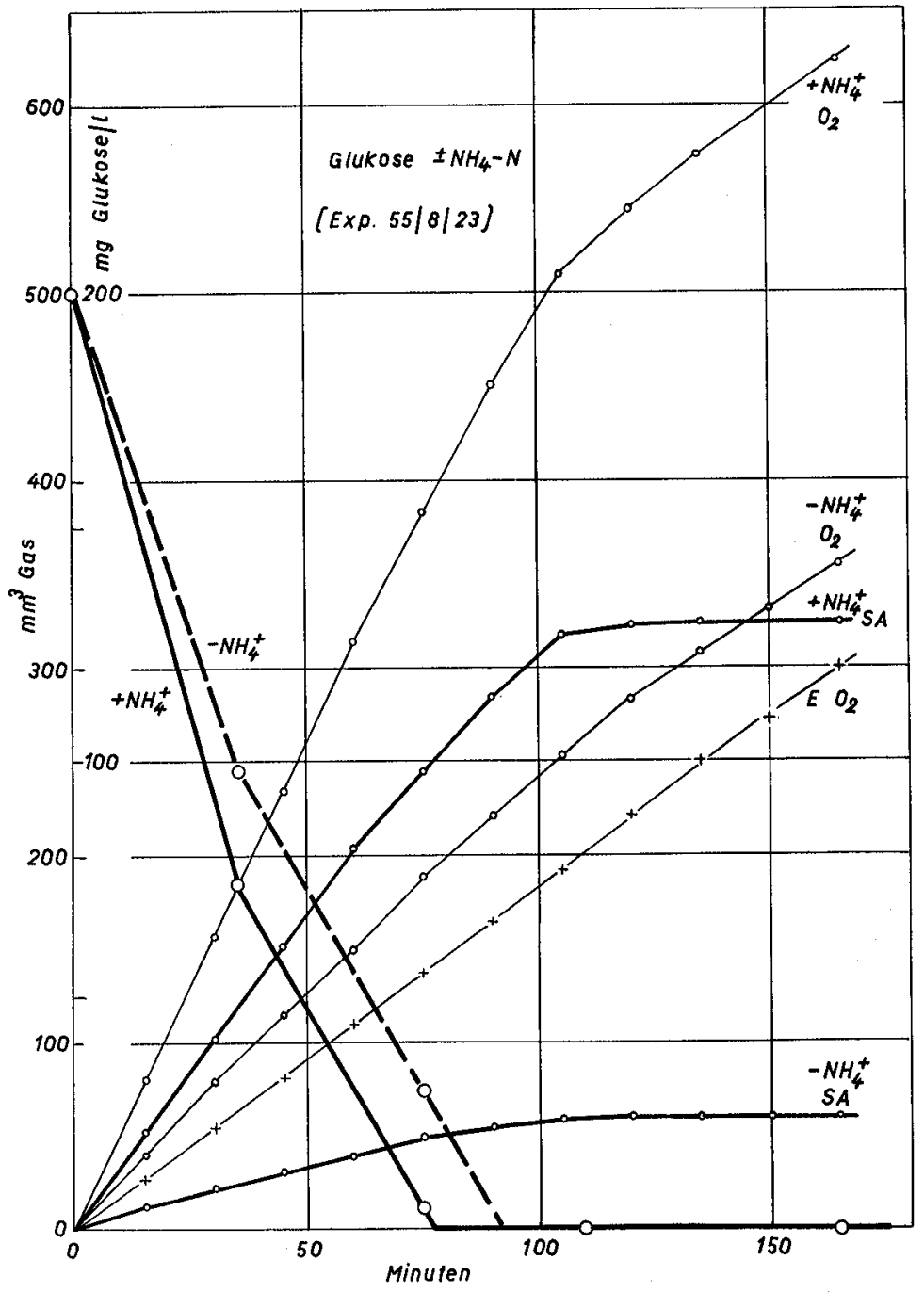

Abbildung 2

Sauerstoffverbrauch von Belebtschlamm während der Elimination von Glukose mit und ohne Gegenwart von Ammonium-Ion. Schlamm nicht an Substrat adaptiert.

Bezeichnungen:

$+\mathrm{NH}_{4}^{+} \mathrm{O}_{2},=$ Substratatmung (Sauerstoffverbrauch) mit und ohne Gegenwart von

$\left.-\mathrm{NH}_{4}^{+} \mathrm{O}_{2}\right\}$ Ammonium-Ion.

$\mathrm{E}_{2}=$ endogene Atmung (Sauerstoffverbrauch).

$\left.\begin{array}{l}+\mathrm{NH}_{4}^{+} \mathrm{SA} \\ -\mathrm{NH}_{4}^{+} \mathrm{SA}\end{array}\right\}=$ zusätzlicher Sauerstoffverbrauch, verursacht durch Substratzusatz.

Kräftig ausgezogene Kurven: Glukosekonzentration in den Respirationsgefässen während des Atmungsversuches. 
eine Stickstoffquelle in Form von $\left(\mathrm{NH}_{4}\right)_{2} \mathrm{SO}_{4}$ zur Verfügung stand, in $\mathrm{Ab}$ bildung 2 zusammengestellt. Dieses Experiment ist in zweierlei Hinsicht aufschlussreich:

In Gegenwart von Ammonium war der $\mathrm{O}_{2}$-Verbrauch bis zur Erschöpfung des Substrates rund $5 \mathrm{mal}$ grösser als in Abwesenheit der N-Quelle.

Die Zuckerresorption erfolgte bei gleichzeitigem Angebot von $\mathrm{NH}_{4}^{+}$ merklich schneller als ohne N-Quelle. Ferner ist die Zeitdifferenz zwischen dem vollständigen Verschwinden des Zuckers aus der Lösung und dem Wiedererreichen der endogenen Atmung des Schlammes bei Gegenwart von $\mathrm{NH}_{4}^{+}$grösser (25 min) als bei Abwesenheit von Ammonium (etwa Io $\mathrm{min}$ ). In beiden Fällen dauert aber die Phase der durch das Substrat verursachten Atmungssteigerung angenähert gleich lang.

Wir haben in Tabelle I die wichtigsten Beobachtungen aus den vor-

Tabelle 1

Auswertung der Experimente 53/5/7, 53/7/3 und 55/8/23 mit Zuckern

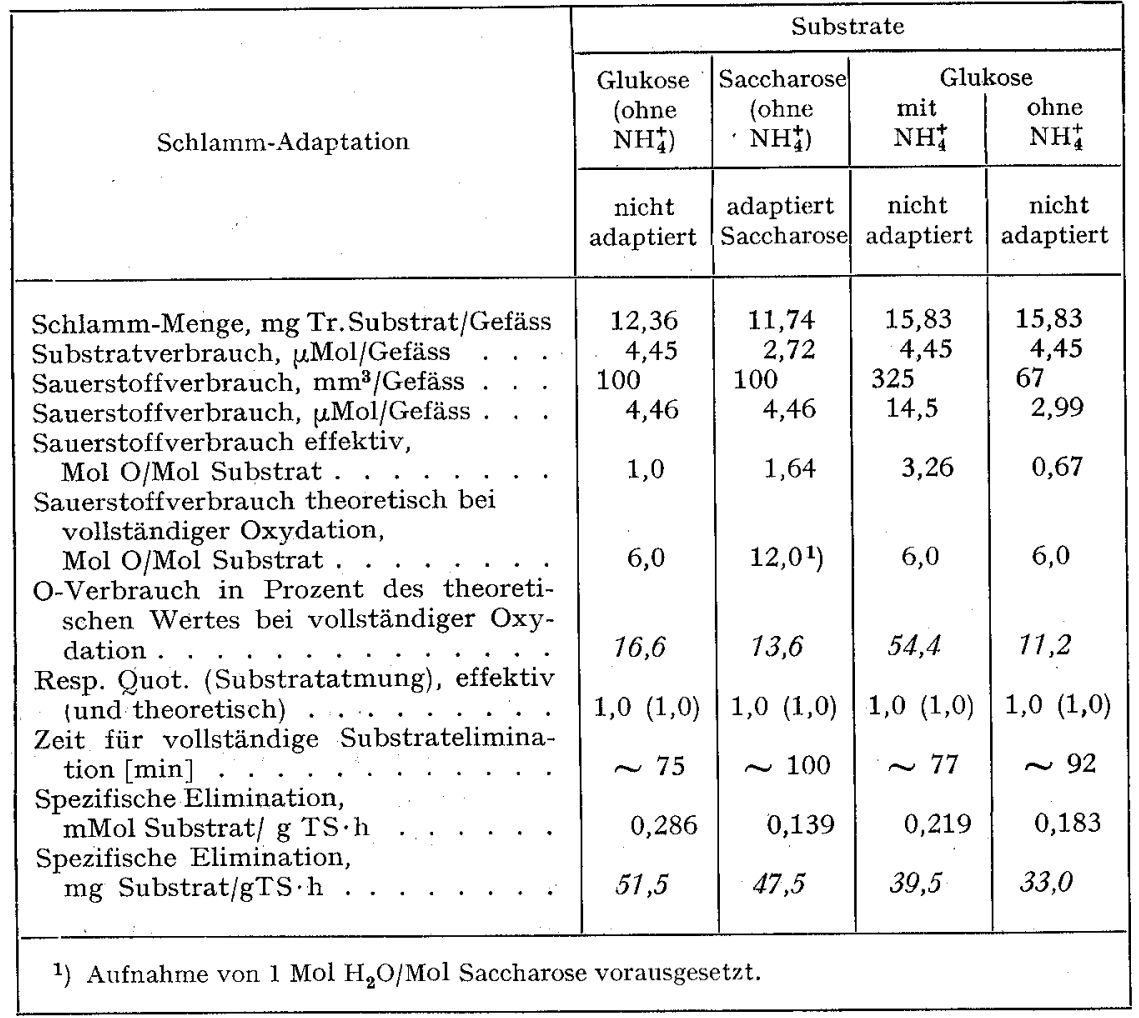


stehend beschriebenen Experimenten zahlenmässig zusammengestellt. In allen Versuchen (und auch in den hier nicht dargestellten Experimenten) ergab sich für die Zuckerveratmung ein Respirationsquotient von $\mathrm{r}, \mathrm{O}$, der genau dem theoretischen Wert bei vollständiger Zerlegung des Zuckers in $\mathrm{CO}_{2}$ und Wasser entspricht. Man darf deshalb aus dem Sauerstoffverbrauch des Schlammes den effektiv veratmeten Anteil des resorbierten Zuckers berechnen und findet, dass in Abwesenheit einer Stickstoffquelle zwischen II und I $6 \%$, bzw. bei Gegenwart von $\mathrm{NH}_{4}^{+}$rund $55 \%$ oxydiert wurden. Es handelt sich dabei natürlich um eine ganz summarische Beurteilung der Gesamtreaktion der komplexen Schlammbiozönose. Das sehr einseitige Verhältnis zwischen veratmetem und oxydativ assimiliertem Zucker in stickstofffreien Lösungen wurde bei Belebtschlamm bereits von PLACAK und Ruchноғт [Io] festgestellt (ihre Methodik erlaubt allerdings keinen direkten Vergleich mit unseren Versuchen). Ähnliche, wenn auch weniger extreme Verhältnisse sind von BARKER [I] bei der Alge Prototheka Zopffi und von Clifton [2] bei Ps. saccharophila gefunden worden (vgl. Zusammenfassung bei CuIfToN $[3,4]$ ). Die beobachtete, starke Verlagerung des Zuckerumsatzes von Assimilation zu Respiration in Gegenwart von Ammonium wurde in ganz ähnlicher Weise auch bei Serratia marcescens (Glyzerol bzw. Zitrat als Substrate) von MCLEAN und FisHer [8] beobachtet. Die Autoren schlossen aus ihren Versuchen, dass die Assimilation des anorganischen $\mathrm{N}$ ein stark endergonischer Prozess sei, der eine vermehrte oxydative Ausnützung der zur Verfügung stehenden Atmungssubstrate zur Folge habe. Eine Reihe anderer Autoren hat diesen Befund seither bestätigt (beispielsweise YemM und FoLKes [I8] bei Torulopsis; SYRETT [I3] bei Cblorella).

Die geschilderten Beobachtungen können wohl nur so verstanden werden, dass die Geschwindigkeit der Konzentrationsabnahme des Zuckers in der Schlammsuspension ausschliesslich von pbysiologiscben Vorgängen bestimmt wird und passive Oberflächeneffekte praktisch bedeutungslos sein müssen. Die Gescbwindigkeit der Resorption des Substrates durch die Mikroflora des Schlammes bestimmt ausscbliesslicb die Geschmindigkeit seiner Elimination. Das Vorauseilen der Elimination gegenüber der Veratmung erklärt sich zwanglos aus dem hohen Anteil assimilierten Substrates. Ein Teil dieser Assimilate besitzt offenbar nur transitorischen Charakter und täuscht noch eine unmittelbare Substratatmung vor, auch wenn das Aussenmilieu bereits vollständig erschöpft ist. Wie weiter unten gezeigt wird, ist bei anderen Substraten diese Erscheinung viel weniger ausgeprägt oder überhaupt nicht vorhanden. 


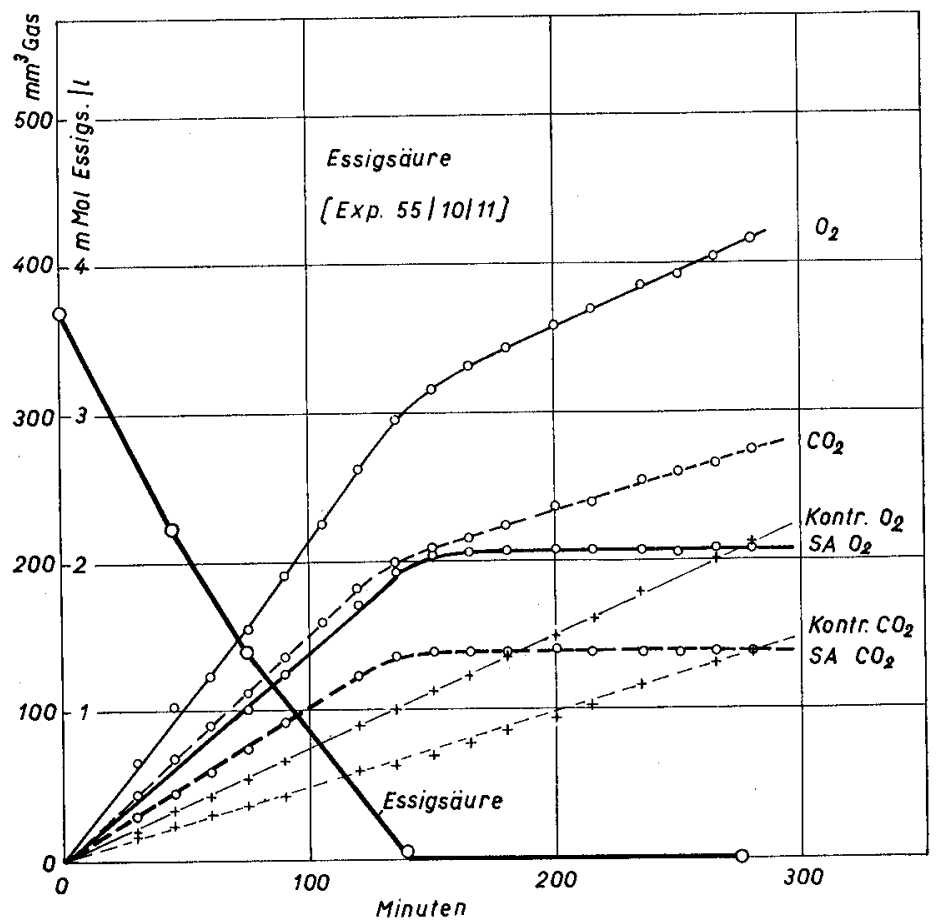

Abbildung $3 \mathrm{a}$

Sauerstoffverbrauch und Kohlensäureproduktion von Belebtschlamm während der Elimination von Essigsäure. Schlamm nicht an Substrate adaptiert. Bezeichnungcn: $O_{2}$ bzw. $\mathrm{CO}_{2}=$ Gasumsatz des Schlammes bei Gegenwart des Substrates (Substratatmung). Kontr. $\mathrm{O}_{2}$ bzw. Kontr. $\mathrm{CO}_{2}=$ Gasumsatz desselben Schlammes ohne Substratzusatz (endogene Atmung). $\mathrm{SA} \mathrm{O}_{2}$ bzw. $\mathrm{SACO} \mathrm{CO}_{2}=$ zusätzlicher Gasumsatz wegen Substratzusatz. Kurven "Essigsäure" bzw. "Buttersäure" = Substratkonzentration im Respirationsgefäss während des Atmungsversuches.

\section{b) Elimination von Monokarbonsäuren}

Als Vertreter eines von den Zuckern stark abweichenden Substrattyps haben wir für weitere Versuche Essigsäure und Buttersäure zugezogen. Wie aus den Darstellungen der beiden Beispiele in Abbildung $3 \mathrm{a}$ und $\mathrm{b}$ hervorgeht, tritt wiederum ein praktisch konstanter Abfall der Substratkonzentration ein. Ferner ist eindeutig, dass beide Säuren ziemlich genau in dem Zeitpunkt in der Aussenlösung verschwinden, in welchem die Substratatmung aufhört. Substratresorption und oxydative Dissimilation fallen also zeitlicls zusammen. Beide weisen annähernd konstante Geschwindigkeit auf. Eine adsorptive Bindung der Säuren an die Schlammflocken ist bei diesen Elektrolyten ebensowenig nachweisbar wie bei den Zuckern. 


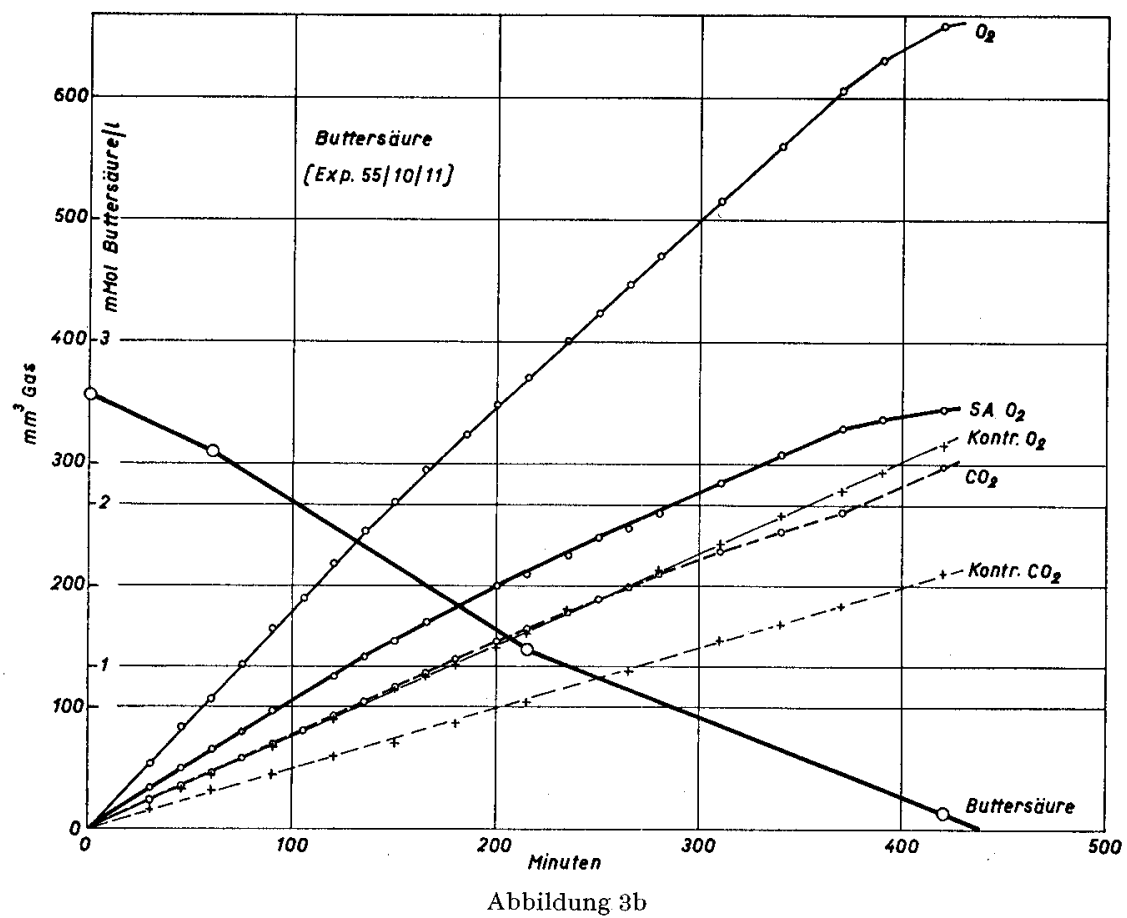

Sauerstoffverbrauch und Kohlensäureproduktion von Belebtschlamm während der Elimination von Buttersäure. Gleicher Schlamm wie bei Versuch von Abb. 3 a. Schlamm nicht an Substrat adaptiert. Bezeichnung vgl, Abbildung 3a.

Wir haben die wichtigsten Daten der beiden Versuchsreihen in Tabelle 2 zusammengefasst. Da beide Experimente gleichzeitig und mit demselben Belebtschlamm vorgenommen wurden, lassen sich die Ergebnisse miteinander vergleichen. Bei beiden Säuren fallen die niedrigen Werte für die Respirationskoeffizienten auf und ebenso der geringe effektive Sauerstoffverbrauch im Verhältnis zur theoretisch benötigten Menge bei vollständiger Veratmung der gebotenen Substrate. Der letztere Effekt ist zweifellos wie bei den Zuckern auf eine stark gesteigerte Assimilation der Säuren im stickstoffarmen Milieu (kein $\mathrm{NH}_{4}^{+}$Zusatz) zurückzuführen. Mit Belebtschlamm, der an die homologen Alkohole der untersuchten Carbonsäuren adaptiert war, fanden MCKINNEY und JERIs [9] Atmungsquoten, die bei Essigsäure $69 \%$, bei Buttersäure $39 \%$ des theoretisch möglichen Wertes betrugen.

Die von den theoretischen Werten stark abweichenden Respirationskoeffizienten lassen darauf schliessen, dass die oxydative Dissimilation der 
Tabelle 2

Auspertung pon Experiment 55/10/11 mit Acetat und Butyrat

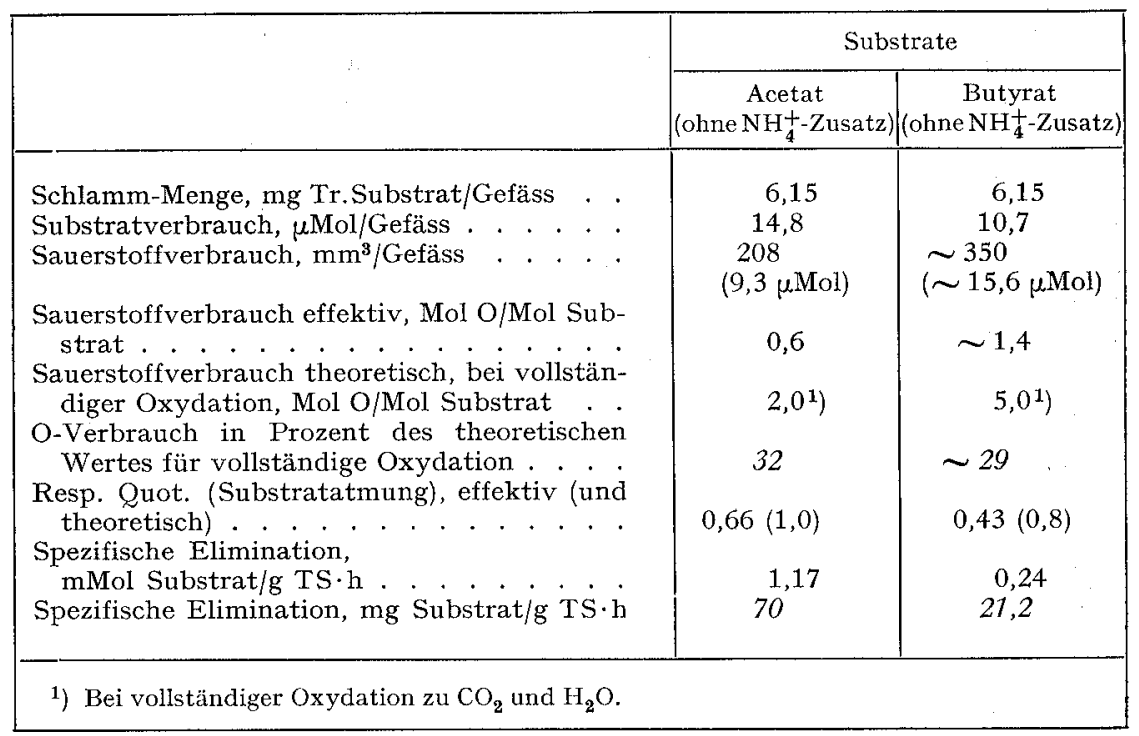

beiden Säuren im Belebtschlammgemisch auf mehreren Wegen erfolgt. Ein direkter Rückschluss auf den Prozentsatz assimilierter Säuren aus dem Sauerstoffverbrauch wie bei den Zuckern ist deshalb anhand der vorliegenden Zahlen ausgeschlossen. In Anbetracht der heterogenen Gärungsflora des Schlammes muss ja wohl auch damit gerechnet werden, dass die vorliegenden Beobachtungen nur der Summe einer Reihe verschiedenster Verarbeitungsmechanismen der gebotenen Substrate Ausdruck geben. Auch konnte bisher noch nicht geprüft werden, ob zum Beispiel bei der Elimination von Buttersäure irgendwelche $Z$ wischenprodukte ihrer Dissimilation gefasst werden können.

Die verhältnismässig geringe Eliminationsgeschwindigkeit der Buttersäure ist übrigens noch von einigem praktischem Interesse, denn es werden auch heute noch für die Reinigung konzentrierter, kohlehydratreicher $\mathrm{Ab}$ wässer (zum Beispiel aus der Zucker- und Stärkeindustrie) sogenannte Gärfaulverfahren empfohlen. Bei diesen Verfahren wird als erste Behandlungsstufe eine anaerobe Gärung vorgenommen, bei welcher in der Regel hohe Buttersäurekonzentrationen entstehen. Erst in einer zweiten Stufe wird dann auf oxydativem Wege die Beseitigung der Gärungssäuren zu erreichen 


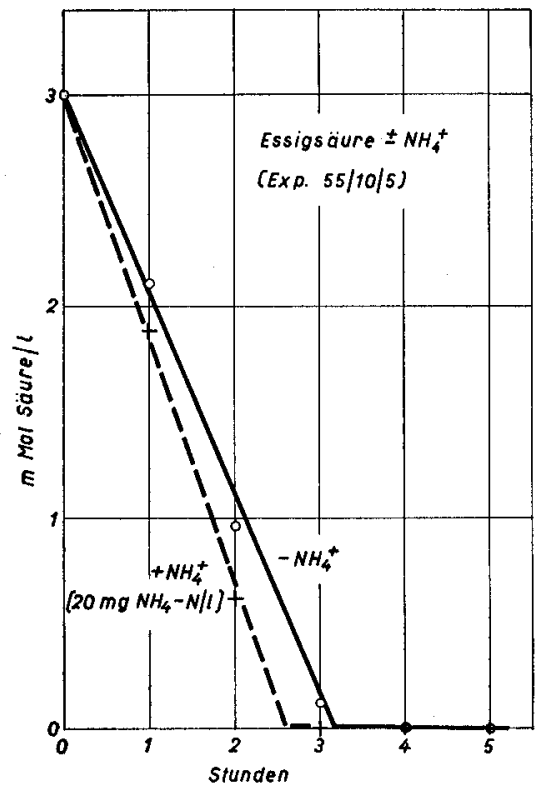

Abbildung $4 \mathrm{a}$

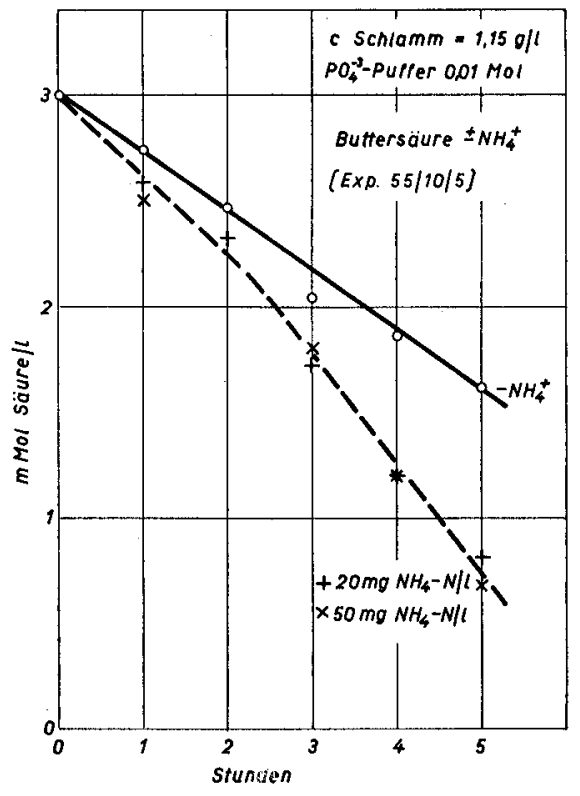

Abbildung 4b

Elimination von Essigsäure (4a) bzw. Buttersäure (4b) durch Belebtschlamm mit und ohne Gegenwart von Ammonium-Ion. Gleicher Schlamm in beiden Versuchen, Schlämme nicht an Substrate adaptiert.

versucht. Vergleicht man die Eliminationsgeschwindigkeiten zum Beispiel von Saccharose (Tabelle I) mit denjenigen von Buttersäure (Tabelle 2), so ist leicht einzusehen, dass dieses zweistufige Verfahren, abgesehen von seinen Unannehmlichkeiten (unvollständige Gärungen wegen starker $\mathrm{pH}-$ Senkung, Geruchsbildung) und seinem grossen Bedarf an Anlagenvolumen, einen unwirtschaftlichen Umweg darstellt.

In ähnlicher Weise wie bei den Zuckern wird auch die Resorption der untersuchten Karbonsäuren durch den Belebtschlamm bei Gegenwart von Ammoniumion in der Lösung beträchtlich beschleunigt, ebenfalls unter starker Atmungssteigerung. Wir geben in den Abbildungen 4a und b zwei Beispiele für die Elimination von Azetat und Butyrat (Makroversuch) wieder, die unter den früher beschriebenen Versuchsanordnungen mit Belebtschlamm aus häuslichem Abwasser gewonnen wurden. Das anfängliche Verhältnis von $3 \mathrm{mM}$ Azetat auf I,43 mM Ammoniumstickstoff beschleunigte die Azetatelimination um das $\mathrm{r}, 3$ fache, bei Butyrat ist unter analogen Verhältnissen die Eliminationsgeschwindigkeit durch die Gegen- 
wart von Ammonium 1,6 mal schneller. Der Versuch mit Butyrat zeigt ferner, dass eine Steigerung des Stickstoff-Säure-Verhältnisses über einen gewissen Grenzwert hinaus keine weitere Beschleunigung der Substratresorption hervorbringt. Es muss allerdings noch durch weitere Versuche abgeklärt werden, welches das niedrigste Verhältnis zwischen Ammonium$\mathrm{N}$ und Kohlenstoffsubstrat ist, das gerade eine maximale Eliminationsgeschwindigkeit des letzteren gewährleistet.

\section{c) Elimination von Aminosäuren}

Wir haben vorläufig nur Versuche mit Asparaginsäure und Tryptophan als Modellsubstanzen vorgenommen, wobei dieselben Methoden wie in den vorstehenden Experimenten verwendet wurden. In den Abbildungen $5 \mathrm{a}$

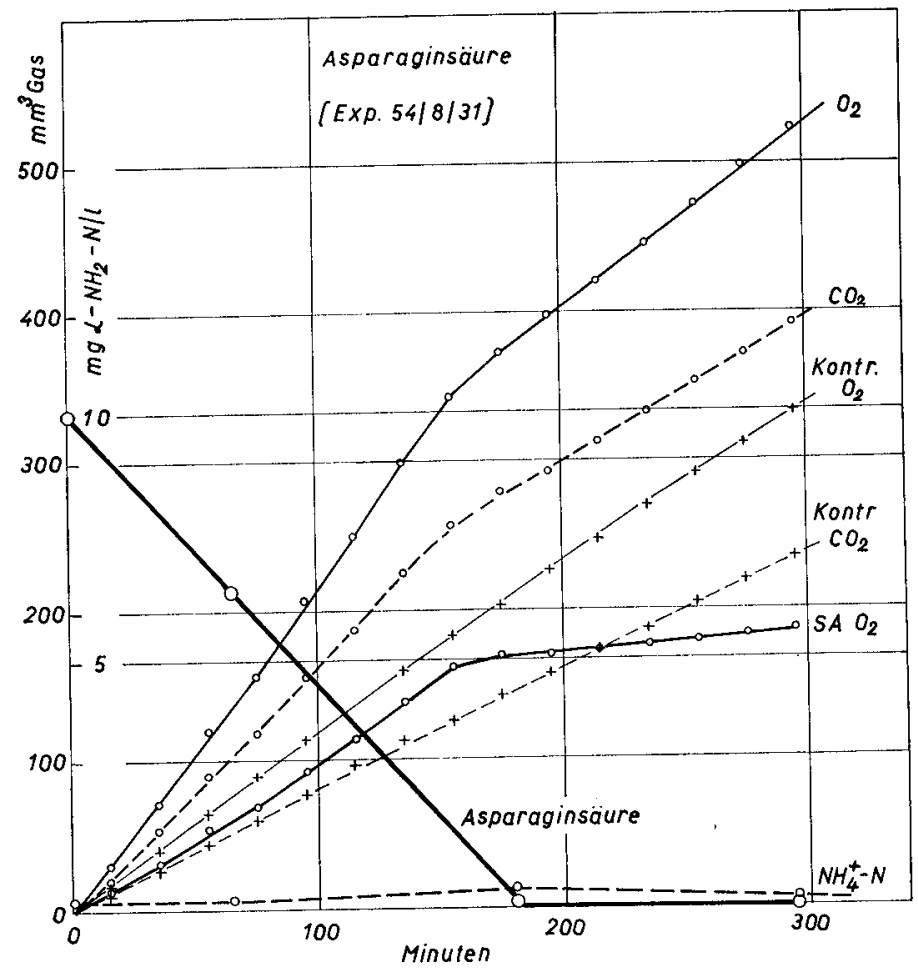

Abbildung 5 a

Sauerstoffverbrauch und Kohlensäureproduktion von Belebtschlamm während der Elimination von Asparaginsäure (5a) bzw. Tryptophan (5b). Gleicher Schlamm in beiden Versuchen, Schlämme nicht an Substrate adaptiert. Bezeichnungen : sinngemäss wie in Abbildung 3 a und $b$. 
und $\mathrm{b}$ sind $z$ wei Parallelversuche dargestellt, die wiederum einen absoluten Vergleich der beiden Substratzusätze erlauben. Es zeigt sich in Übereinstimmung mit früheren Beobachtungen, dass ein annähernd linearer Konzentrationsabfall der beiden Substrate erfolgt (wenigstens innerhalb der analytisch zugänglichen Konzentrationsbereiche). Bei beiden Substraten (vor allem aber bei Tryptophan) ist aus der Sauerstoffaufnahmekurve eine ausgeprägte Adaptation des Schlammes festzustellen, die insofern überrascht, als man annehmen kann, dass diese Aminosäuren in der gewohnten

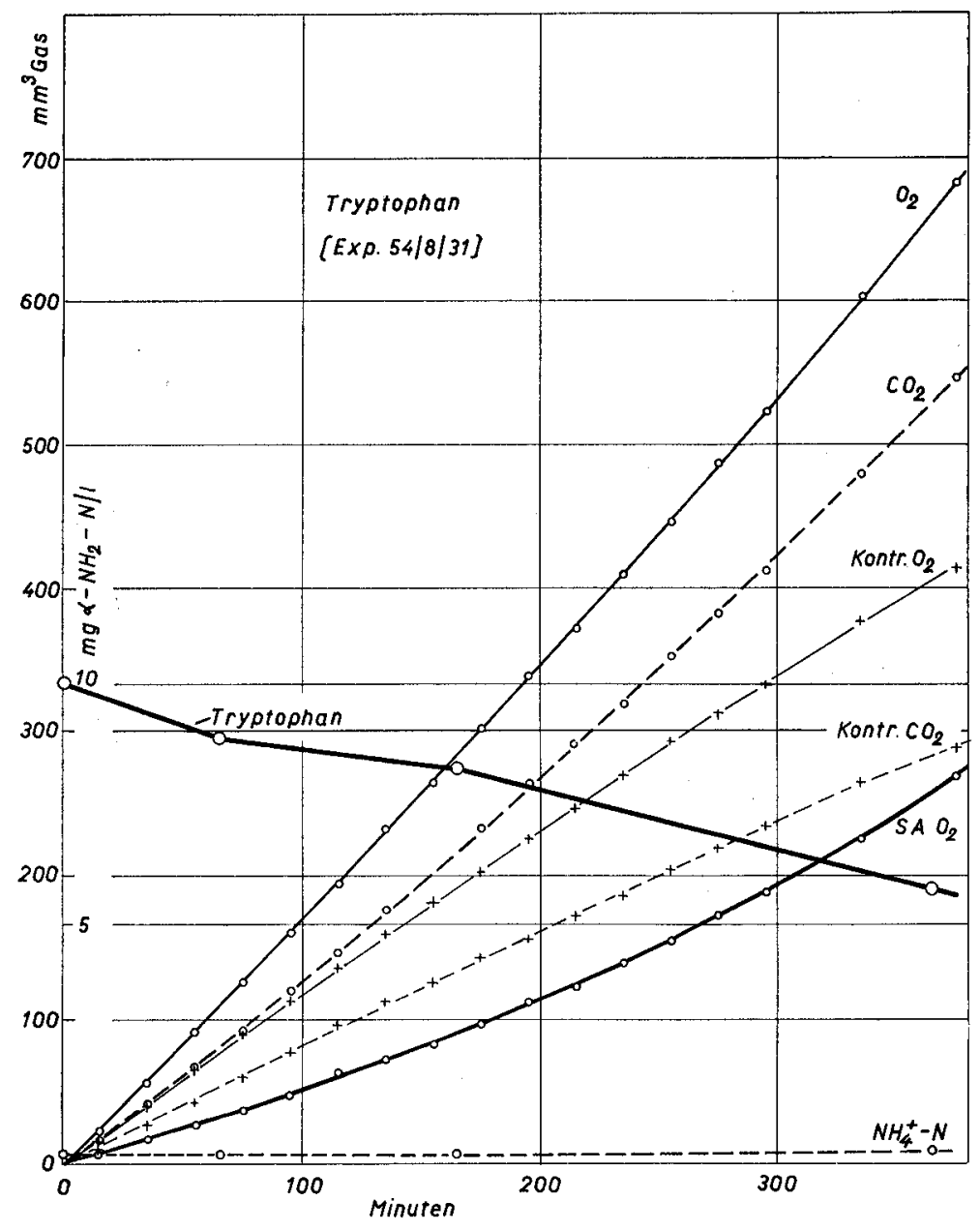

Abbildung 5b

Erklärung vgl. Abbildung $5 \mathrm{a}$ 
Tabelle 3

Auswertung von Experiment 54/8/31 mit Asparaginsäure und Tryptopban

\begin{tabular}{|c|c|c|}
\hline & \multicolumn{2}{|c|}{ Substrat } \\
\hline & Asparaginsäure & Tryptophan \\
\hline 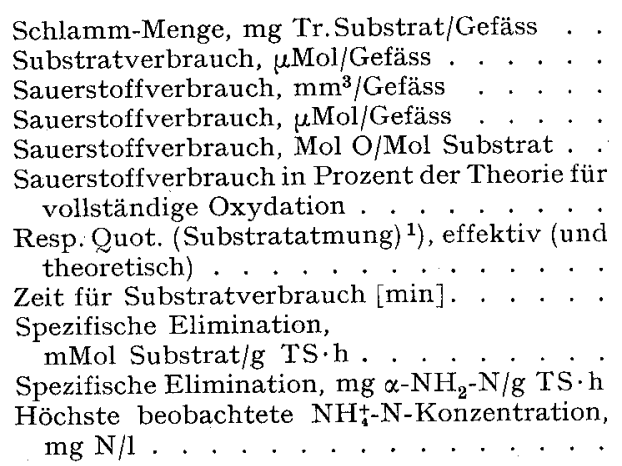 & $\begin{array}{c}13,61 \\
2,86 \\
184 \\
8,21 \\
2,87 \text { (theor. } 3,0) \\
95,6 \\
0,75(0,75) \\
182 \\
0,0694 \\
0,97 \\
\left.0,42(0,20)^{2}\right)\end{array}$ & $\begin{array}{c}13,61 \\
1,22 \\
269 \\
12,0 \\
9,85(\text { theor. } 12,5) \\
78,8 \\
0,73 \rightarrow 0,80(0,88 \\
375 \\
0,0143 \\
0,2 \\
\left.0,30(0,20)^{2}\right)\end{array}$ \\
\hline $\begin{array}{l}\text { 1) Ohne Berücksichtigung der Oxydation der NH } \\
\text { 2) } 0,20=\text { Anfangswert im Schlammwasser vor S }\end{array}$ & $\begin{array}{l}\text { uppe und des Imid } \\
\text { ratzusatz. }\end{array}$ & lazol-N. \\
\hline
\end{tabular}

Nährlösung des Schlammes, das heisst dem Abwasser, stets vorhanden sind. Die Adaptationserscheinung ist wohl nur eine Folge der Heterogenität des Gärungssystems, indem in unseren Versuchen, dank der relativ hohen, vorgelegten Aminosäurekonzentrationen, sich eine viel grössere Zahl von Bakterienarten des Schlammes am Abbau beteiligen konnte als unter den Verhältnissen in einer Kläranlage, wo die absolute Konzentration dieser Substrate ausserordentlich gering ist [16]. Aus Tabelle 3 geht - in auffälligem Gegensatz zu den Beobachtungen bei Zuckern und Karbonsäuren (Tabellen I und 2) - hervor, dass der Sauerstoffverbrauch für den oxydativen Abbau der beiden Aminosäuren sehr nahe an den theoretischen Wert für vollständige Oxydation herankommt und gleichzeitig nur ein Bruchteil des Aminosäurestickstoffs, der von der Desaminierung der gebotenen Substratmenge erwartet werden müsste, in der Lösung als Ammonium erscheint. Bei Asparaginsäure wurde in der Periode der Substratatmung ein $r_{q}$ gefunden, der genau dem theoretischen Wert entspricht, und auch bei Tryptophan ergibt sich im Laufe der Versuchszeit eine starke Annäherung an den Respirationskoeffizienten für vollständige Oxydation des Substrates. Die Beurtei- 
lung der O-Bilanzen und der Respirationskoeffizienten würde deshalb den Schluss zulassen, dass das Kohlenstoffskelett der beiden. AS zu einem wesentlichen Teil vollständig veratmet wird und eine oxydative Assimilation nennenswerter Mengen der beiden Aminosäuren - im Gegensatz zu den untersuchten Kohlenstoffsubstraten -- nicht eintritt. Ob diese Schlussfolgerung - so gut sie durch die Beobachtungen scheinbar gestützt wird im einzelnen richtig ist, muss allerdings angesichts des heterogenen biologischen Systems einer Belebtschlammbiozönose offenbleiben. Im Rahmen dieser Untersuchung scheint uns vor allem das Ergebnis wichtig, dass bei der Elimination der untersuchten Aminosäuren (und sehr wahrscheinlich auch anderer Vertreter dieser Verbindungsklasse) ein Sauerstoffverbrauch eintritt, welcher rechnungsmässig ihrer vollständigen Oxydation entspricht (gegenüber $13-50 \%$ bei Zuckern und rund $30 \%$ bei Karbonsäuren).

Es ist noch von Interesse, die absoluten Eliminationsgeschwindigkeiten der Aminosäuren im Lichte der bisher zum Beispiel in häuslichen Abwässern gefundenen Konzentrationen dieser Verbindungen zu betrachten. Wir haben früher angegeben [16], dass im Abwasser der Stadt Zürich eine Gesamtkonzentration von rund $0,4-0,6 \mathrm{mg} \alpha$-Aminostickstoff/l in gelöster Form (ohne vorgängige Hydrolyse) nachzuweisen ist. Diese Gesamtmenge verteilt sich natürlich auf alle in den menschlichen Abgängen enthaltenen Aminosäuren, so dass auf einzelne Vertreter wohl nur Mengen in der Grössenordnung von wenigen bis vielleicht maximal $30-50 \mu \mathrm{g} \alpha-\mathrm{NH}_{2}$ $\mathrm{N} / \mathrm{l}$ entfallen. Die in Experiment 55/8/3I (Tabelle 3 ) angegebenen spezifischen Eliminationen für Asparaginsäure und Tryptophan lassen leicht erkennen, dass diese Verbindungen in kürzester Zeit (wenigen Minuten) aus dem einem Belebtschlamm zugesetzten Abwasser entfernt sein sollten, wenn ein ähnlich intensiver Kontakt zwischen Schlammorganismen und Abwasser in einer Reinigungsanlage erzielt wird, wie er in den Manometerversuchen vorhanden ist.

\section{Diskussion der Ergebnisse}

Die beschriebenen Experimente lassen wiederum nur den einen Schluss zu, dass die Elimination der untersuchten organischen Verbindungen aus der wässerigen Lösung durch den Belebtschlamm ausschliesslich pbysiologischen Reaktionen der beteiligten Organismen zu verdanken ist und rein chemisch-physikalische Vorgänge (Adsorption) höchstens eine untergeordnete Bedeutung haben können. Der direkt messbare Vorgang der Konzentrationsabnahme eines Substrates entspricht seiner Resorption durch die 
lebenden Zellen des Schlammes. Die anschliessende intrazelluläre Weiterverarbeitung der resorbierten Substratmolekeln - oxydative Assimilation oder Veratmung - lässt sich in den heterogenen Gärungsbiozönosen nur summarisch verfolgen und ist für das Endresultat der gesamten Leistung des Systems auch nicht von Bedeutung. Ein gewisser Einblick in die Stufen der Assimilation und Dissimilation ist allerdings zur Beurteilung der Sauerstoffmenge notwendig, die dem Belebtschlamm zur Verfügung gestellt werden muss. Auf diesen Punkt werden wir in einer späteren Arbeit speziell zurückkommen.

Die Tatsache, dass die Resorption durch Organismen massgebend ist für die Geschwindigkeit, mit welcher die Konzentration einer organischen Substanzin einem Belebtschlamm-Abwasser-Gemisch abnimmt, stellt dieeinzigartige Bedeutung heraus, die der freien Zelloberfläche in einem Belebtschlamm für die Intensität der Abwasserreinigung zukommt. Als «freie Zelloberfläche» verstehen wir diejenige, die sich direkt in Kontakt mit der Lösung der zu eliminierenden Stoffe, das heisst mit dem Abwasser befindet. Die mikroskopische Betrachtung eines Belebtschlammes zeigt - und dies ist ein charakteristisches Merkmal dieser Biozönose -, dass der grösste Teil der Bakterienzellen in grössern oder kleinern, zoogloenartigen Aggregationen zusammengeballt ist. Die freie Zelloberfläche im vorstehenden Sinne umfasst deshalb nur einen kleinen Bruchteil der theoretisch möglichen, so dass der grösste Teil der Substratmolekeln beträchtliche Diffusionswege zurückzulegen hat, bis sie die physiologisch aktiven Zellgrenzflächen erreichen. Es ist unzweifelhaft, dass die aus den vorstehenden Experimenten und in unserer ersten Arbeit berechneten Eliminationsgeschwindigkeiten jedenfalls nicht Maximalwerten entsprechen, denn bei keiner der gewählten Versuchsanordnungen wurde eine Zerteilung des Belebtschlammes erreicht, die über jene hinausging, die auch in einem gut gebauten Belüftungsbecken erzielt werden kann. Man muss deshalb annehmen, dass noch eine erhebliche Steigerung der Eliminationsgeschwindigkeit gelöster Stoffe aus einer Lösung zu erreichen wäre, wenn der Belebtschlamm zu einer annähernd monozellulären Dispersion aufgeteilt werden könnte. In welchem Umfange dies zum Beispiel durch eine Steigerung der Turbulenz mittels mechanischer Mittel möglich ist, kann mangels experimenteller Erfahrungen zur Zeit nicht beurteilt werden. Es entzieht sich auch unserer Kenntnis, ob der dafür benötigte Kraftaufwand in einem vernünftigen Verhältnis zur Steigerung der Eliminationsleistung eines Belebtschlammsystems stehen würde. Eine Abklärung dieser Frage würde sich zweifellos lohnen, und zwar speziell im Hinblick auf die Reinigung konzentrierter industrieller Abwässer. 
Die Assimilation eines Teils der gebotenen Substrate führte in unseren Versuchen im Laufe der Beobachtungsperiode wohl zu einem gewissen Scblammzumacbs, der sich in einer allmählichen Steigerung der Eliminationsgeschwindigkeit (bei konstanter spezifischer Elimination) hätte zeigen sollen. Dies gilt speziell für die Experimente, in welchen sowohl eine Kohlenstoff- als auch eine Stickstoffquelle vorgelegt wurde (Zucker- und Karbonsäureversuche mit $\mathrm{NH}_{4}^{+}$, Aminosäureversuche). Eine uiberschlägige Berechnung zeigt aber, dass dieser Schlammzuwachs im Moment der vollständigen Substratelimination höchstens zwischen 5 und $10 \%$ der ursprünglichen organischen Substanz des Schlammes erreichen konnte. Diese Zunahme der Organismenmasse vermochte sich bei der Eliminationsgeschwindigkeit des Substrates nicht bemerkbar zu machen, zeigte sich aber zum Beispiel beim Aspartatversuch eindeutig darin, dass nach Veratmung der Aminosäure die endogene Atmung grösser war als beim Kontrollschlamm (vgl. Abbildung 5 a). Dieselbe Beobachtung gilt auch für den in Abbildung 2 dargestellten Glukose- $\mathrm{NH}_{4}^{+}$-Versuch.

\section{Zusammenfassung}

I. Die Konzentrationsveränderung von organischen Substraten (Zuckern, Karbonsäuren, Aminosäuren) nach ihrem Zusatz zu Belebtschlamm wurde gleichzeitig mit dem Gasstoffwechsel der Schlämme bestimmt (mikromanometrische Methode).

2. Es konnte gezeigt werden, dass die Resorption von Zuckern durch den Schlamm schneller verläuft als die Veratmung bzw. oxydative Assimilation. Bei Karbonsäuren und Asparaginsäure ist die Veratmung des Substrates ungefähr gleichzeitig mit seinem vollständigen Verschwinden aus der Lösung beendet.

3. Die Experimente zeigen, dass die Elimination der untersuchten organischen Elektrolyte und Nichtelektrolyte in gleicher Weise ausscbliessich durch physiologische Reaktionen der Schlammorganismen erfolgt und chemischphysikalische Prozesse (zum Beispiel Adsorption) dabei keine erkennbare Rolle spielen.

4. Die Anteile der veratmeten und oxydativ assimilierten Mengen der resorbierten Substrate konnten teilweise bestimmt werden. In Übereinstimmung mit Untersuchungen an Reinkulturen von Mikroorganismen wird auch beim Belebtschlamm die oxydative Assimilation einer Kohlenstoffquelle zugunsten der Respiration zurückgedrängt, wenn der Schlamm gleichzeitig Ammonium-Stickstoff assimilieren kann. 
5. Durch die Gegenwart von $\mathrm{NH}_{4}^{+}$-Stickstoff wird die Resorption der untersuchten Kohlenstoffverbindungen merklich beschleunigt.

6. Es wird die Schlussfolgerung gezogen, dass der Dispersion eines Schlammes und damit der Turbulenz in einem Belüftungsbecken, eine entscheidende Bedeutung für die Geschwindigkeit der Elimination eines Substrates zukommt.

\section{SUMMARY}

(r) The rate of concentration decrease of various substrates (sugars, fatty acids, amino acids) after addition to suspensions of activated sludge was estimated simultaneously with the oxygen and $\mathrm{CO}_{2}$ metabolism of the sludge (micromanometric method).

(2) It was demonstrated that the resorption of sugars by sludge occurs at a higher rate than the respiration and oxydative assimilation of these substrates. Substrate respiration with fatty acids and amino acids is terminated however simultaneously with the complete elimination of the substrates from the sludge suspensions.

(3) It is concluded that the physiological reactions, measured as 'elimination' of both the electrolytes and non electrolytes used as substrates, is identical with the resorption of the subtrate molecules by the sludge organisms and that physicochemical reactions such as adsorption are of no practical significance.

(4) In certain instances the substrate proportions respired and oxydatively assimilated could be measured. In accordance with pure cultures of microorganisms the oxydative assimilation of a carbon source by activated sludge is reduced in favour of respiration when a nitrogen source such as ammonia can be assimilated simultaneously.

(5) In presence of ammonia as a nitrogen source the resorption of the carbon compounds is significantly accelerated.

(6) It is concluded that turbulence in the aeration bassins of activated sludge plants $s$ a limiting factor for the rate of the elimination of substrates by sludge.

\section{LITERATURVERZEICHNIS}

[I] BARker, H. A., J. cell. comp. Physiol. 8, 23 I (r936).

[2] Clifton, C. E., J. Bact. 33, r45 (I937).

[3] Cilifton, C. E., Adv. Enzymol. 6, 269 (1946).

[4] Clufton, C. E., in: Bacterial Pbysiology (New York I95I).

[5] DrXon, M., Manometric Methods (Cambridge 1943).

[6] Gellman, J., und Heukelekian, H., Sewage Industr. Wastes 25, i I96 (I953).

[7] Hoover, S. R., JAsewicz, L., und Porges, N., Sewage Industr. Wastes 25, I 163 (1953). 
[8] MCLEan, D. C., und Fisher, K. C., J. Bact. 54, 599 (1947).

[9] MCKiney, R. E., und Jeris, J. S., Sewage Industr. Wastes 27, 728 (1955).

[io] Placak, O. R., und Ruchioft, C. C., Publ. Health Rep. 62, 697 (i947).

[II] Ridenour, G. M., Sewage Wks. J. 7, 25 (I935).

[i2] Ruchhoft, C. C., Kachmar, J. F., und Moore, W. A., Publ. Health Rep. 55, 393 (I940).

[i3] SYRETT, P. J., Ann. Bot. 17, I, 2 r (1953).

[14] Thériault, E. J., Sewage Wks. J. 7, 377 (1935).

[is] Thértault, E. J., Ind. Eng. Chem. 28, 83 (1936).

[I6] WuhrmanN, K., Verh. int. Ver. theor. angew. Limnol. 10, 580 (1949).

[77] Wuhrmann, K., v. Beust, F., und Ghose, T. K., Schweiz. Z. Hydrol. 2o, 284 (I958).

[18] YеMM, E. W., und Folkes, B. F., Biochem. J. 57, 495 (I954). 